\title{
SuperK-Gd: The Gd future of Super-Kamiokande
}

\author{
Lluís Martí-Magro* on behalf of the Super-Kamiokande Collaboration \\ Kamioka Observatory, Institute for Cosmic Ray Research, University of Tokyo. \\ E-mail: martilluesuketto.icrr.u-tokyo.ac.jp
}

\begin{abstract}
Water Cherenkov detectors are notable for their large masses and their charge particle detection capabilities. These two properties have been exploited in depth in many searches with outstanding results at Super-Kamiokande. On the other hand, neutrons are usually captured on protons and produce a single $2.2 \mathrm{MeV}$ gamma. The Compton scattered electron that is being produced does not yield enough Cherenkov light for the PMT coverage (40\%) of Super-Kamiokande to be efficiently detected. However, detecting neutrons would be an important improvement in most of our analyses to either improve our signal event selection (for processes that produce neutrons) or background event rejection (for processes that do not produce any neutron). Gadolinium (Gd) has the largest thermal neutron capture cross section. In this process, it emits a gamma cascade with a total energy of about $8 \mathrm{MeV}$ which would be possible to detect with high efficiency. The EGADS project demonstrated that it is possible to safely add Gd to Super-Kamiokande and the collaboration approved in June 2015 the SuperK-Gd project. The necessary refurbishment of the Super-Kamiokande detector started in June 2018 and finished in early 2019. In this talk, we will report about this work and the project outlook.
\end{abstract}

36th International Cosmic Ray Conference -ICRC2019-

July 24th - August 1st, 2019

Madison, WI, U.S.A.

${ }^{*}$ Speaker. 


\section{Introduction}

Water Cherenkov detectors are based on a very well established and mature technology. In a nutshell, when a charged particle moves in a medium faster than light in that medium, a cone of electromagnetic radiation is emitted. Depending on the transparency of the medium, this light can traverse relatively long distances and then be then detected by a photomultiplier tube (PMT). Super-Kamiokande (SK) consists of a stainless steel tank of about $40 \mathrm{~m}$ in height and diameter that contains 50 kton of ultra-pure water. A well adjusted water purification system constantly removes impurities in the water and ensures a good water transparency. Thus, when a charged particle is travelling through the SK tank with sufficient energy, it produces Cherenkov light and most of this light reach the PMTs and are detected. The detection of this light is done with about 11,000 PMTs in the inner detector (ID) and allows the reconstruction of the interaction point and the energy of the event. Ultra-pure water is relatively easy and cheap to maintain and the intrinsic backgrounds relatively low. SK is located about $1 \mathrm{~km}$ underground under the Mt. Ikenoyama and the muon flux is reduced to about $6 \times 10^{-8} \mathrm{~cm}^{-2} \mathrm{~s}^{-1} \mathrm{sr}^{-1}$.

\section{Physics}

SK is a versatile detector with many studies in the neutrino sector. SK can detect neutrinos from about 3.5 MeV to $100 \mathrm{TeV}$. Some of the most important sources are the neutrinos from nuclear fusion in the Sun's core, the neutrinos from the interactions of high energy cosmic rays with the Earth's atmosphere, neutrinos from accelerators, neutrinos from beta decays in nuclear reactors, and neutrinos from core-collapse supernovae (and if we get really lucky, from our galaxy or neighbourhood). Because of the many protons it contains, SK also looks for proton decay.

Neutral particles cannot be seen directly at SK. One important example are neutrons. Proton decay, if it happens, is then a very rare event and SK has the best limits. A prototypical example is $p \rightarrow e^{+} \pi^{0}$. Very few neutrons are expected in a proton decay event. On the other hand, the main background are atmospheric neutrinos, which often produce at least one neutron.

A core-collapse supernova ( $\mathrm{SN}$ ) in our galaxy is a rare event. The duration of a $\mathrm{SN}$ burst is on the order of 10 seconds and of the vast amount of energy released $~ 99 \%$ goes into neutrinos. The last (and the first too) SN whose neutrinos have been detected and unequivocally linked to the SN was 1987a [1]. The flux of a single SN burst far from our galaxy is not large enough to be detected but in the history of the universe there have been many $\mathrm{SNe}$, creating a copious, ubiquitous and isotropic neutrino flux: the diffuse SN neutrino background (DSNB). These neutrinos mainly interact though inverse beta decay (IBD) $\bar{v}_{e}+p \rightarrow n+e^{+}$. Because of (so far) irreducible backgrounds, the DSNB has not been observed yet. Again, SK has the best limits [2] and if neutrons could be efficiently detected these backgrounds could be greatly reduced and make their detection possible for the first time.

Thus, by gaining more information about the final state of a reaction through the efficient detection of neutrons, we would both achieve the efficient rejection of background events and the selection of signal events in many studies at SK.

To accomplish an efficient neutron detection, GADZOOKS! proposed to dissolve Gd [3]. Gadolinium (Gd) has one of the highest cross sections for thermal neutron capture (49,000 barns) 
where the isotopes with the largest cross sections are ${ }^{157} \mathrm{Gd}$ and ${ }^{155} \mathrm{Gd}(\sim 255,000$ and $\sim 61,000$ barns respectively), and natural abundances $15.65 \%$ and $14.80 \%$. When a neutron is captured on ${ }^{157} \mathrm{Gd}$ or ${ }^{155} \mathrm{Gd}$ a gamma cascade of total energy $7.9 \mathrm{MeV}$ or $8.5 \mathrm{MeV}$, respectively, follows. Most of the energy is carried by 3-4 gammas, with an energy equivalent to an electron of about 4-5 $\mathrm{MeV}$ total energy. As a result, these neutron captures can be efficiently detected at SK. When a neutron is produced in ultra-pure water, for example in IBD $\bar{v}_{e}+p \rightarrow n+e^{+}$or spallation events, these neutrons are first thermalised and then mostly captured on free protons (cross section on free protons is 0.3 barns while on oxygen it is 0.19 milibarns) after about $200 \mu \mathrm{s}$. After the capture a single $2.2 \mathrm{MeV}$ gamma is then produced. This gamma is then mostly detected through Compton scattering by an electron, which cannot be efficiently detected given the photon energy and PMT photo-coverage at SK.

$\mathrm{Gd}$ is a rare-earth metal that can be dissolved in water in form of a salt. The best compound we have tested is Gd sulfate which is easily soluble and has optimal optical properties. In fact, the easiest to dissolve is $\mathrm{Gd}$ sulfate in the octahydrated form: $\mathrm{Gd}_{2}\left(\mathrm{SO}_{4}\right)_{3} \cdot 8 \mathrm{H}_{2} \mathrm{O}$ (8 molecules of water per Gd atom). Hereafter, we will refer to it as Gd sulfate only.

Dissolving Gd sulfate in the otherwise ultra-pure water at SK quickly increases the fraction of neutron captures on Gd up to a concentration of about $0.02 \%$ of Gd sulfate in mass, see Figure 1. At this concentration, $50 \%$ of the neutron captures are on Gd. From this concentration on more $\mathrm{Gd}$ sulfate has to be dissolved than before to gain a similar fraction of captures on $\mathrm{Gd}$ (the sign of the curvature changes from convex to concave). A concentration ten times larger, $0.2 \%$ of $\mathrm{Gd}$ sulfate in mass, brings the fraction of captures on Gd to $90 \%$. From here on increasing the number of captures on Gd is increasingly difficult. Our final target at SK is to achieve $90 \%$ of the captures on $\mathrm{Gd}$, i.e. a Gd sulfate concentration of $0.2 \%$ of Gd sulfate in mass.

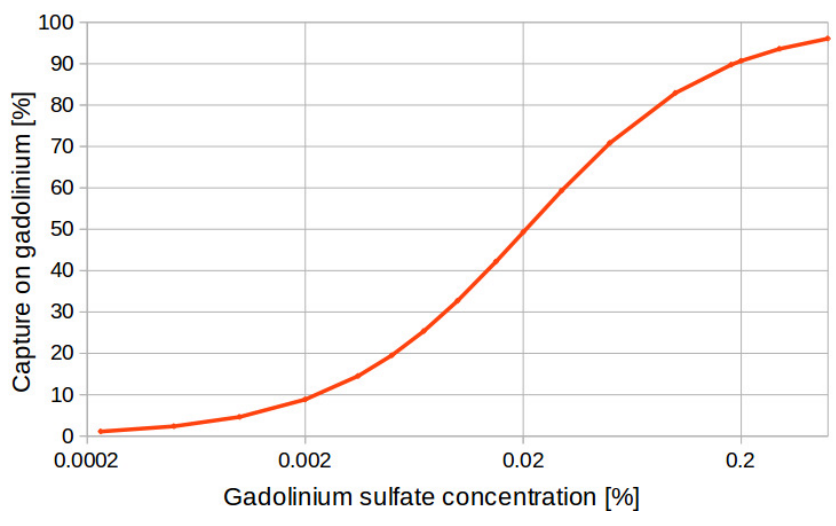

Figure 1: Fraction of neutrons captured on $\mathrm{Gd}$ as a function of Gd sulfate concentration in water by mass.

\section{EGADS}

SK started taking data in 1996. Two years later, the SK Collaboration announced the first evidence of neutrino oscillations. After the proposal to dissolve Gd in SK in the late 2002, the development of the necessary tools to carry out the idea and the needed tests started at the University of California, Irvine (UCI). It was decided that before introducing Gd into SK a full scale test 
was needed first. Hence, in 2009 the EGADS R\&D project (Evaluating Gadolinium's Action on Detector Systems) was funded for a 200-ton class WC detector test and further development. A new cavern near the SK site was excavated to accommodate this new facility. At its start, EGADS featured a 200-ton stainless steel tank, a Gd sulfate dissolving and pre-treatment system, a water purification system and a device to measure the water transparency, UDEAL (Underground Device Evaluating Attenuation Length). All the installed materials at this point and later that are in contact with water are made with the same materials as SK was made.

The EGADS project had to attain five objectives before being ready for SuperK-Gd:

- Show that the purification system can achieve and maintain good water quality while keeping the Gd concentration constant.

- Show that Gd sulfate has no negative effects on detector components.

- Study how to reduce the now visible neutron background from U/Th fission chains in Gd sulfate impurities, ambient neutrons, etc.

- Demonstrate that it will not negatively affect other SK analyses through transparency loss or increase of backgrounds.

- Prove that Gd can be added/removed in an efficient and economical way.

The main results from EGADS will be summarized here in this section.

The Cherenkov light left after travelling $15 \mathrm{~m}$ (LL15) is shown in Figure 2, upper data points. Since this is the typical distance that Cherenkov photons travel in SK, it is the best estimation of the water transparency. The blue band represents the typical LL15 values during SK-III and SK-IV and the blue, red and green data points are the three sampling positions in the EGADS tank: bottom, centre and top, respectively. The vertical grey shaded bands represent changes in the running conditions in time. For example, the four first lines are the $\mathrm{Gd}$ sulfate loading until the final concentration of $0.2 \%$ in mass. We see the same pattern for each loading: during the Gd loading LL15 decreases and then rapidly increases again until it recovers (back to the blue band). The other grey vertical bands represent changes in the water system running conditions: yearly maintenance, and water systems tests (turning on/off parts of the water system to investigate its effects). In these cases we see a similar situation where LL15 decreases and recovers once the usual conditions are restored. The conclusion from the above is that the purification system can achieve and maintain good water quality and that Gd sulfate is basically transparent to Cherenkov light once is diluted.

Figure 2 also shows the concentration of Gd sulfate in EGADS, lower set of data points. The concentration increases in all sampling points after each Gd loading and after the last one it stays constant within the measurement uncertainties (the values are around the horizontal dashed line that indicates the expected final concentration). The main lessons are that the Gd concentration is homogeneous and that while the water quality is comparable to that of SK, the Gd losses are not detectable, i.e. the concentration is constant.

After this period, the EGADS tank was drained. For this task we used a special resin that removes $\mathrm{Gd}$, avoiding $\mathrm{Gd}$ to be released to the environment even if there is no official regulation regarding Gd-loaded water. This procedure had been previously tested with sample measurements 


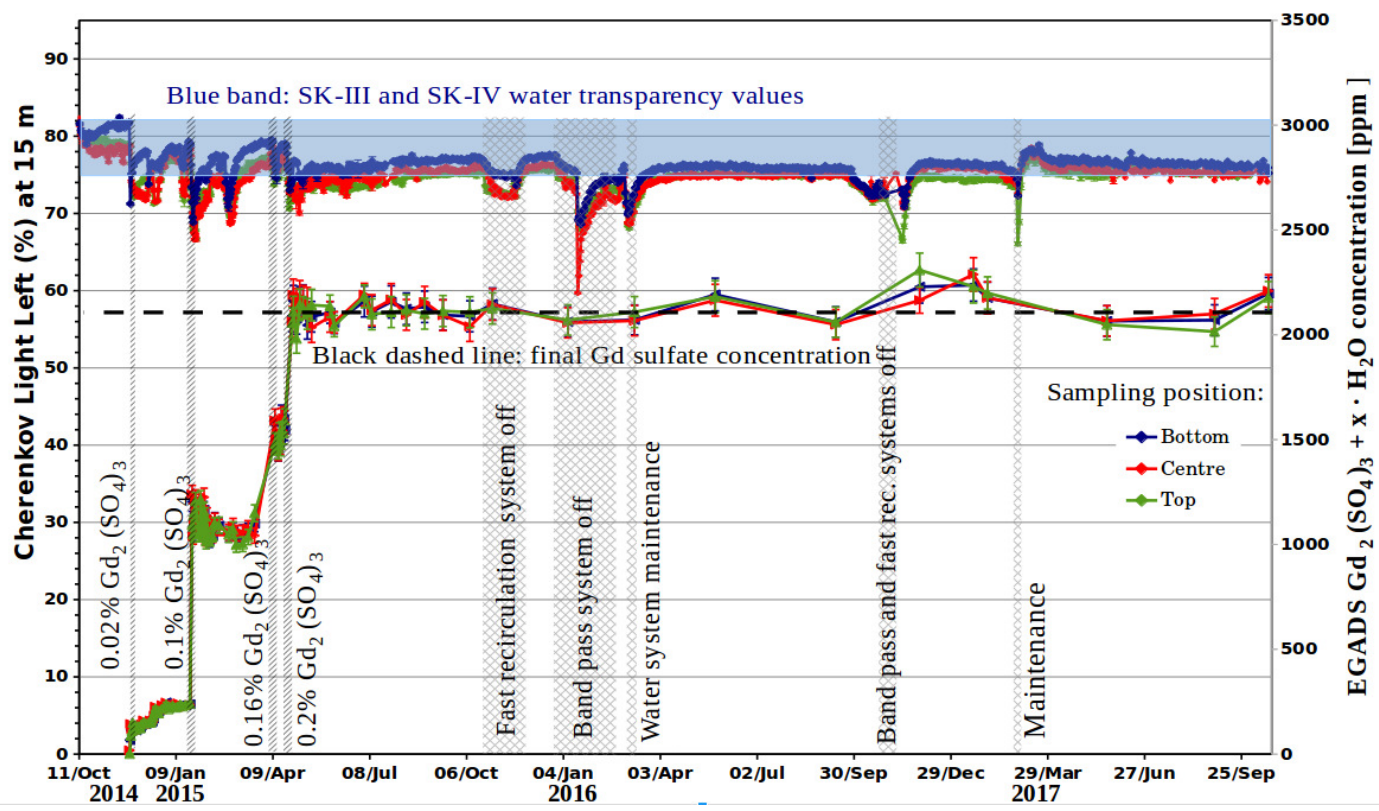

Figure 2: Cherenvov light left after traveling $15 \mathrm{~m}$, upper data points. The blue band represents the typical LL15 values during SK-III and SK-IV. The blue, red and green data points are the three sampling positions in the EGADS tank: bottom, centre and top, respectively. The vertical grey shaded bands represent changes in the running conditions in time. The concentration of Gd sulfate in EGADS is also shown, lower set of data points, where the horizontal dashed line that indicates the expected final concentration.

using an ICP/MS. Since we could not find any trace of Gd, we could only set limits on the concentration and concluded that it is $<5 \mathrm{ppb}$. Because of the good Gd-removal efficiency of this method and the acceptable costs when scaling it to SK, we chose it. In fact, a facility for the Gd removal in SK has been already installed in the SK mine.

Once the EGADS tank was empty we proceeded to the inspection of the tank structure, PMTs and other detector components and evaluate the effects of a prolonged soaking in the most similar conditions ever done before for SK. We found no adverse effects as it was in fact expected from the good LL15 values.

Now that efficient neutron tagging is possible, the neutron background will become visible. It is specially important to quantify the impurities in Gd itself. As in the case of Gd, those impurities would be homogeneously dissolved in the detector too (unlike neutrons coming from the interaction of muons with the mine rock surrounding the SK tank). Hence, we conducted studies to determine our radio-purity requirements. The most stringent constrains come from Solar and DSNB analyses since they can mimic their signals, see Table 1. Only the most restrictive values are shown ("-" for the less restrictive).

A collaboration with several companies, where they produce Gd sulfate and we test the radiopurities of their samples was established. For this purpose, we used low background germanium detectors at Canfranc (Spain) and Boulby (UK) and ICP/MS measurements in Kamioka (Japan). Within our experimental uncertainties, one of the companies met our requirements. They are currently producing the first Gd sulfate for loading SK.

Because the water transparency and the backgrounds are under control, the current analyses in 


\begin{tabular}{c|c|c|cc} 
Chain & $\begin{array}{c}\text { Part of the } \\
\text { chain }\end{array}$ & $\begin{array}{c}\text { Typical } \\
(\mathrm{mBq} / \mathrm{Kg})\end{array}$ & $\begin{array}{c}\text { DSNB } \\
(\mathrm{mBq} / \mathrm{Kg})\end{array}$ & $\begin{array}{c}\text { Solar } \\
(\mathrm{mBq} / \mathrm{Kg})\end{array}$ \\
\hline${ }^{238} \mathrm{U}$ & ${ }^{238} \mathrm{U}$ & 50 & $<5$ & - \\
& ${ }^{226} \mathrm{Ra}$ & 5 & - & $<0.5$ \\
\hline${ }^{232} \mathrm{Th}$ & ${ }^{232} \mathrm{Th}$ & 10 & - & $<0.05$ \\
& ${ }^{228} \mathrm{Th}$ & 100 & - & $<0.05$ \\
\hline${ }^{235} \mathrm{U}$ & ${ }^{235} \mathrm{U}$ & 32 & - & $<3$ \\
& ${ }^{227} \mathrm{Ac} /{ }^{227} \mathrm{Th}$ & 300 & - & $<3$ \\
\hline \hline
\end{tabular}

Table 1: Radioactive contamination and typical impurities in untreated Gd sulfate and our requirements from DSNB and Solar neutrino analyses.

SK should not be affected.

\section{SK refurbishment}

As shown above, the EGADS goals have been met and thus, on June $27^{\text {th }} 2015$ the SK Collaboration decided to move forward and approve the SuperK-Gd project. Soon a new cavern near the SK tank was excavated to install a new water system for Gd-loaded water. This new water system has been designed according to the experience in EGADS and large parts of the equipment has been already installed.

Although SK looks like a cylinder, when viewed from above, SK is an icosagon. SK is made of 400 plates of stainless steel $(6 \mathrm{~m} \mathrm{x} 2 \mathrm{~m} \mathrm{x} 4 \mathrm{~mm})$ which are welded together. The welding length is of the other of several kilometres long and besides this, there are thousands of bolts that penetrate these plates. It is not difficult to imagine that there could be, even if small, a leak in the tank. In fact, a leak of had been found and estimated to be of about 1 ton/day

Hence, in addition to the aforementioned goals, the leak in the SK tank had to be fixed. The candidate material for this task had to meet several requirements. Mechanically it should be soft, flexible and capable of standing an external stretching force. It should not elute into water and posses a good long-term stability in water. Similarly to Gd sulfate, it should be a material with low radioactivity. Many materials were tested and finally one was found.

The SK tank was opened in May 31st 2018 to finally start the refurbishment work. The main tasks that were carried out were:

- Clean the walls and structures to remove dust and exhaust gas from the construction work in 1994-95. Remove potential sources of rust. Forgotten tools and other non-stainless steel pieces were found and the stainless steel had to be cleaned and recovered.

- About 140 newly developed PMTs 50-cm for Hyper-Kamiokande were installed in the inner detector (ID). As shown in figure 3, these new PMT [4] have a higher quantum efficiency.

- In the outer detector (OD), about 200 8-inch PMTs were also replaced. After the replacement of ID and OD PMTs the new tubes were tested. When this had to be done, the day before 
after the refurbishment work, the tank was closed and next day before the work would start again, the signal of those new PMTs were tested.

- Covering the whole surface of the OD, reflective white Tyvek ensure a high detection efficiency of muons. The old Tyvek sheets were removed and new ones were installed.

- The original piping in SK had never been modified. The circulation rate at the end SK-IV was $60 \mathrm{~m}^{3} / \mathrm{h}$ and there was little room to modify the flow in the ID and the OD independently, see Figure 4 left. During the refurbishment work, the piping was modified to increase the water flow to $120 \mathrm{~m}^{3} / \mathrm{h}$ and to allow for different flow configurations inside the tank, see Figure 4 right.

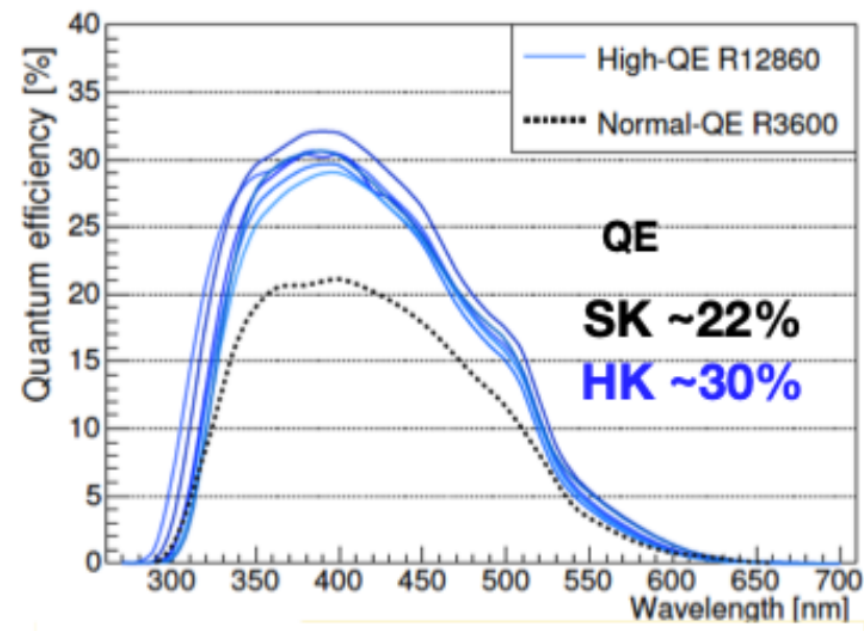

Figure 3: Comparison of the quantum efficiencies of a typical SK PMT and the new Hyper-Kamiokande (HK) model.
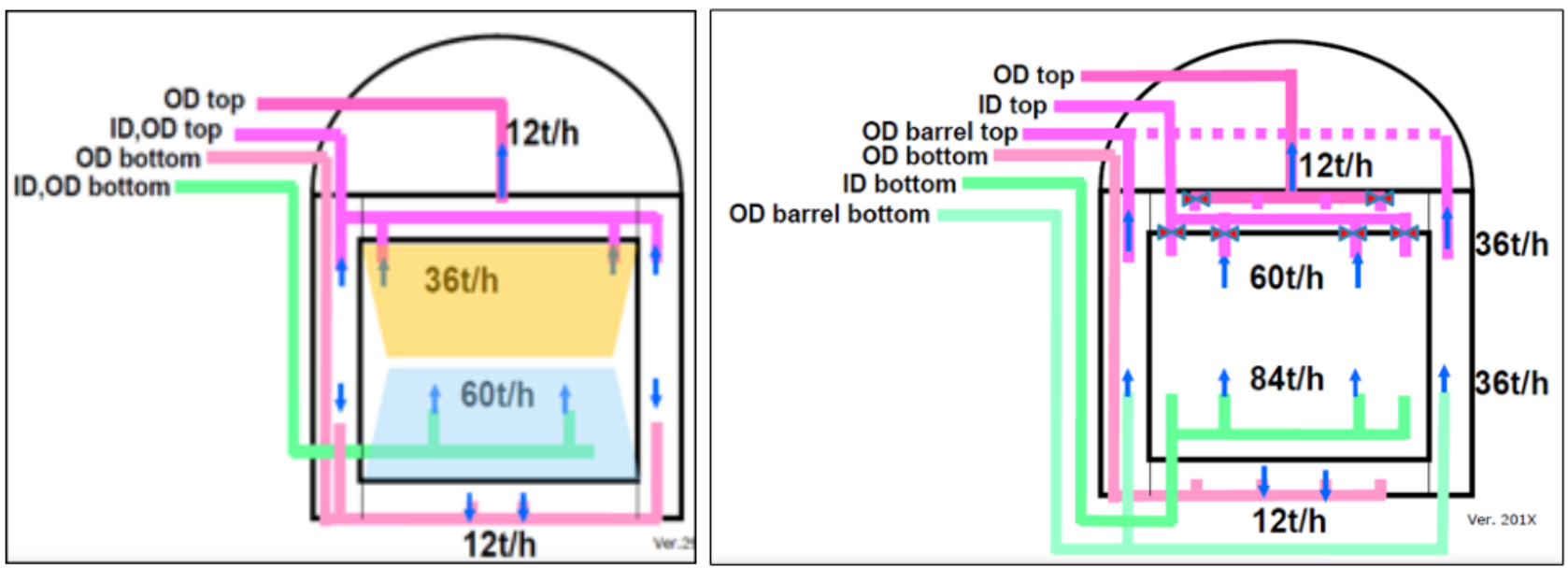

Figure 4: Previous (left) and new (right) piping schemes at SK. 
To recover the water quality in SK as soon as possible, the new water system is being operated as an ordinary water purification system (similar as in previous phases of SK). As can be seen in Figure 5, the current water quality is good.

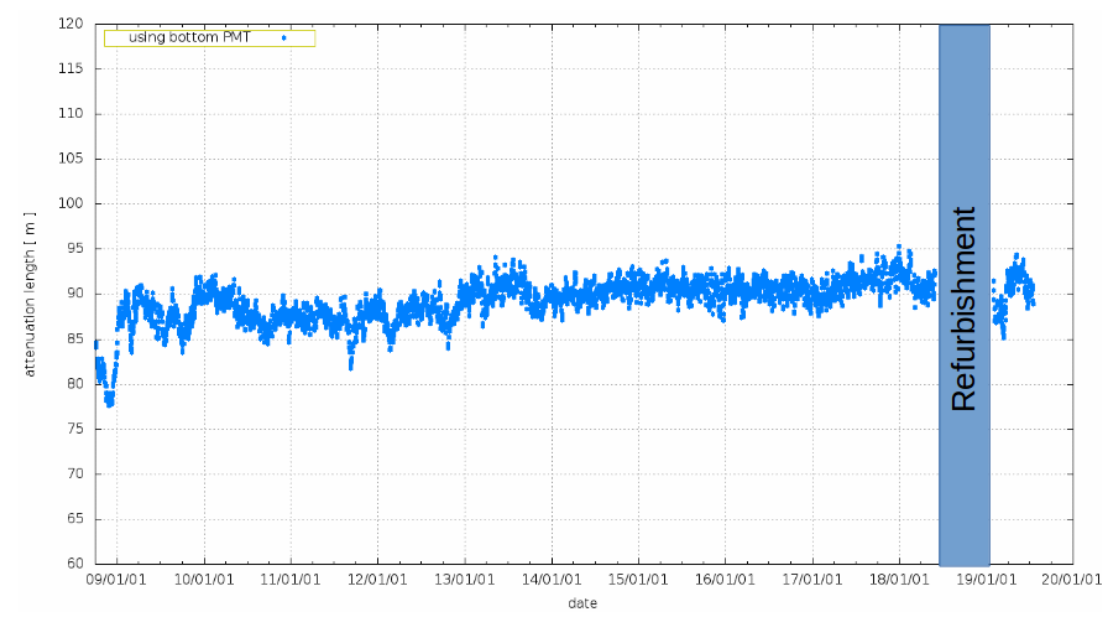

Figure 5: Water transparency since 2009 (SK-IV) until now (SK-V). The water transparency in SK-V has recovered quickly and is now at a similar level to that of SK-IV.

\section{References}

[1] K. Hirata et al. [Kamiokande-II Collaboration], Phys. Rev. Lett. 58, 1490 (1987). doi:10.1103/PhysRevLett.58.1490

[2] K. Bays et al. [Super-Kamiokande Collaboration], Phys. Rev. D 85 (2012) 052007 doi:10.1103/PhysRevD.85.052007 [arXiv:1111.5031 [hep-ex]].

[3] J. F. Beacom and M. R. Vagins, "GADZOOKS! Anti-neutrino spectroscopy with large water Cherenkov detectors,” Phys. Rev. Lett. 93, 171101 (2004) doi:10.1103/PhysRevLett.93.171101 [hep-ph/0309300].

[4] [Hyper-Kamiokande Collaboration], KEK-PREPRINT-2016-21, ICRR-REPORT-701-2016-1. 